\title{
Scintillating scotoma following optic nerve compression caused by an unruptured anterior communicating artery aneurysm: a case report
}

\author{
Megumi Matsuda ${ }^{1,2^{*}}$ (D), Takeo Murahashi ${ }^{1}$, Yusuke Nakagaki ${ }^{1}$, Hideki Endo ${ }^{2}$ and Youichi Nakagaki
}

\begin{abstract}
Background: Scintillating scotoma caused by optic nerve compression by aneurysm is rare. We report a case of scintillating scotoma following optic nerve compression caused by an unruptured anterior communicating artery aneurysm.
\end{abstract}

Case presentation: A 64-year-old woman visited our department with a scintillating scotoma. Magnetic resonance imaging demonstrated no new lesions, although her previously diagnosed anterior communicating artery aneurysm had increased in size. Thus, we performed neck clipping. The operative view showed that the aneurysm compressed the right optic nerve. Her symptoms disappeared after the operation. These findings suggest that scintillating scotoma may be caused by compression of the optic nerve by the anterior communicating artery aneurysm.

Conclusion: Although scintillating scotoma is a common condition, aneurysms should be considered as a potential cause.

Keywords: Anterior communicating artery aneurysm, Scintillating scotoma, Optic nerve compression, Optic neuropathy

\section{Background}

Scintillating scotoma is commonly associated with migraines and is not a characteristic symptom of optic nerve compression. Indeed, to our knowledge, there are no reports of scintillating scotoma caused by optic nerve compression. Furthermore, anterior communicating artery (AcomA) aneurysms rarely present with symptoms of compression of the anterior visual pathways [1]. Herein, we report a case of scintillating scotoma following optic nerve compression caused by an unruptured AcomA aneurysm.

\footnotetext{
*Correspondence: mamemimumo.dan106@gmail.com

1 Department of Neurosurgery, Takikawa Neurosurgical Hospital, 1-2-5,

Nishimachi, Takikawa, Hokkaido 073-0044, Japan

Full list of author information is available at the end of the article
}

\section{Case presentation}

A 64-year-old woman with a family history of subarachnoid hemorrhage presented to an ophthalmology department with problems with her vision. Her symptoms were suggestive of a scintillating scotoma, which involved a crescent-shaped flickering light of zigzag fortification on the right inferior quadrant of the visual field for a duration of $1 \mathrm{~h}$. The symptoms occurred twice over a few days. She had no medical history of migraine and no headache associated with the scintillating scotoma. Fundus examination showed no remarkable changes of the optic disk of both eye and no vision loss. The patient then visited our hospital for intracranial examination. She had no headache or neurological deficit. Magnetic resonance imaging showed no new lesions that could have caused her visual symptoms, including no infarction or intracranial carotid artery stenosis. Magnetic resonance angiography showed increased growth of a previously diagnosed unruptured AcomA aneurysm compared with 5 years prior. Catheter 
angiography was performed. Angiogram indicated that the maximum aneurysm diameter was $5.1 \mathrm{~mm}$ with formation of aneurysm blebs (Fig. 1). We scheduled neck clipping, which involved a left-sided frontotemporal craniotomy for microsurgical clip ligation of the aneurysm. Intraoperative findings included no clot around the aneurysm, indicating that the aneurism was unruptured. The aneurysm dome sat deeply into the right optic nerve, causing nerve compression (Fig. 2a). After detaching the aneurism, the optic nerve showed a concave section at the aneurysm attachment site (Fig. 2b). Following the operation, the scintillating scotoma disappeared and no visual field deficit was detected. The patient was discharged from our hospital with no neurological deficit.

\section{Discussion}

We present a case of scintillating scotoma with an unruptured AcomA aneurysm. Scintillating scotoma is commonly associated with migraines, with a potential mechanism involving cortical spreading depression [2]. Cortical spreading depression is a slowly propagating wave of neuronal and glial depolarization. Shams et al. also reported several cases of migraine-like visual aura secondary to cerebral lesions, including infarction, arteriovenous malformations, and tumors [3]. In that study, the lesions were located in the occipital lobe, which contains the primary visual cortex. The propagation of spreading depression through the visual cortex will create similar visual phenomena, whatever the original cause [3]. In our

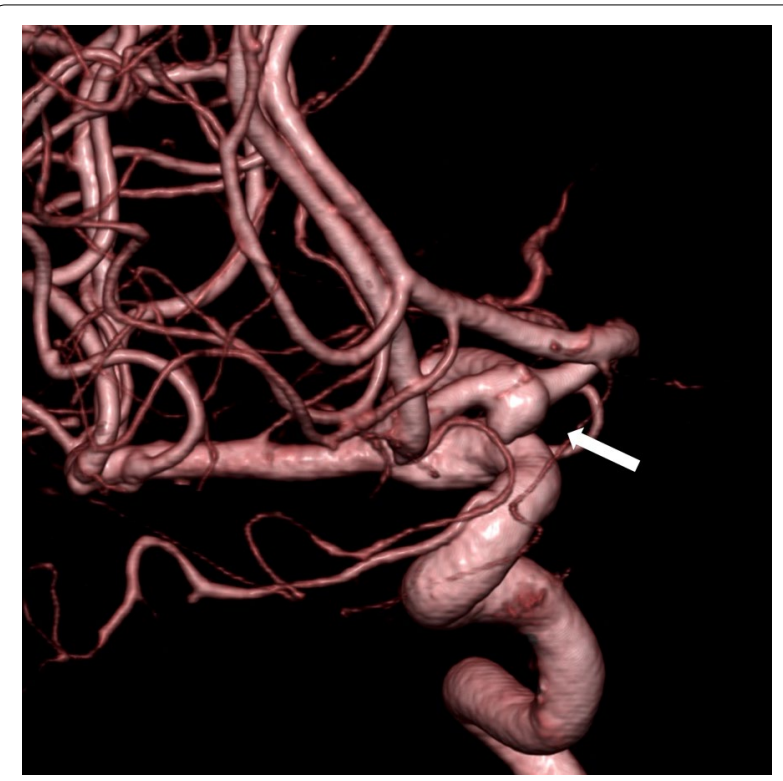

Fig. 1 Angiogram showing that the anterior communicating artery aneurysm had a maximum diameter of $5.1 \mathrm{~mm}$ with formation of blebs (arrow)

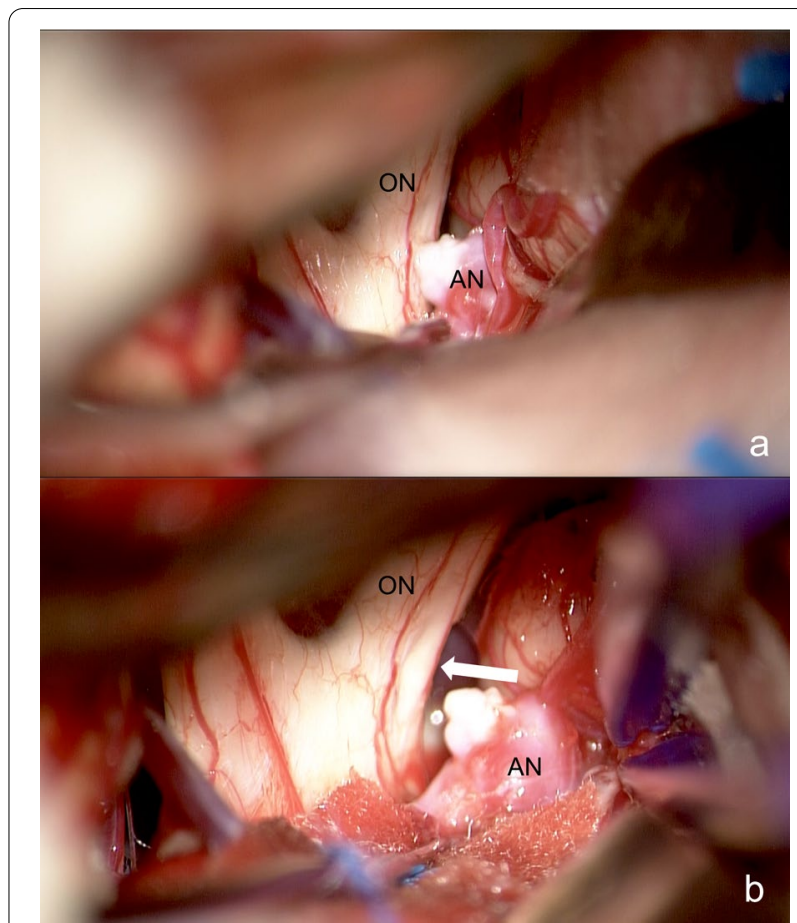

Fig. 2 a Surgical photograph showing compression of the optic nerve by the aneurysm dome. The aneurysm dome sat deeply into the right optic nerve. $\mathbf{b}$ After detaching the aneurism, the optic nerve showed a concave section at the aneurysm attachment site (arrow). AN, aneurysm; ON, optic nerve

patient, the aneurysm compressed the right optic nerve. To our knowledge, there are no previous reports of scintillating scotoma caused by optic nerve compression, including that it is caused by brain lesions such as tumors or aneurysms. Nevertheless, optic nerve compression is likely to cause scintillating scotoma as the optic nerve is a key visual pathway. Furthermore, Hoshina et al. reported a case of optic neuritis mimicking migraine with scintillating scotoma [4]. Thus, scintillating scotoma may be a type of optic neuropathy.

AcomA aneurysms rarely present with symptoms of compression of the anterior visual pathways [1]. In general, AcomA aneurysms tend to rupture, while they are relatively small and cause visual symptoms. When the aneurysm ruptures, pressure from both the arterial blood flow and the hematoma may compress the optic nerve, which may cause visual symptoms [5]. Lee et al. reported a 60-year-old man who presented with acute monocular vision loss following subarachnoid hemorrhage from a ruptured AcomA aneurysm [6]. By contrast, there are limited case reports of visual symptoms associated with unruptured AcomA aneurysms. Shukla et. al reported a 65-year-old patient presenting with visual loss, complete loss of vision in the right eye, and temporal hemianopsia 
in the left eye caused by a giant $(26 \times 25 \times 23 \mathrm{~mm})$ AcomA aneurysm [1]; the aneurysm was large enough to compress the anterior visual pathways. To our knowledge, there are no reports of compression of the anterior visual pathways by small unruptured AcomA aneurysms. Our patient had a small and unruptured aneurysm, although it showed formation of blebs, which were heading toward the optic nerve. The aneurism had also increased in size, which may have caused progressive optic nerve compression. Finally, our patient showed occasional scintillating scotoma, which may have been caused by aneurysm pressure as well as poor blood circulation to the optic nerve related to enlargement of the aneurysm sac. Based on the operative finding of optic nerve compression by the aneurysm and the disappearance of the visual symptoms after clipping, the scintillating scotoma may have been caused by optic nerve compression by the AcomA aneurysm.

\section{Conclusions}

Neurosurgeons or ophthalmologists frequently see patients with scintillating scotoma. In most patients, this is caused by migraines, although some cases are caused by cerebral lesions. Compression of the optic nerve or optic chiasm by an aneurysm may also cause scintillating scotoma. Thus, aneurysms should be considered in patients showing persistent scintillating scotoma without a headache or medical history of migraine, or when migrainous symptoms are different from prior episodes.

\section{Abbreviations}

AcomA: Anterior communicating artery.

\section{Acknowledgements \\ We thank Edanz (https://jp.edanz.com/ac) for editing a draft of this manuscript.}

\section{Authors' contributions}

All authors contributed to the study conception and design. Material preparation, data collection, and analysis were performed by Megumi Matsuda. The first draft of the manuscript was written by Megumi Matsuda, and all authors commented to previous version of the manuscript. All authors read and approved the final manuscript.

\section{Funding}

The authors have not received any funding for the case study.

\section{Declarations}

Ethics approval and consent to participate

Approval was obtained from the ethics committee of Takikawa Neurosurgical Hospital.

\section{Consent for publication}

The patient has consented to the submission of the case report to the journal.

\section{Competing interests}

The authors declare that they have no competing interest.

\section{Author details}

${ }^{1}$ Department of Neurosurgery, Takikawa Neurosurgical Hospital, 1-2-5, Nishimachi, Takikawa, Hokkaido 073-0044, Japan. ${ }^{2}$ Department of Neurosurgery,

Nakamura Memorial Hospital, Hokkaido, Japan.

Received: 23 November 2021 Accepted: 29 January 2022

Published online: 07 March 2022

\section{References}

1 Shukla DP, Bhat DI, Devi BI. Anterior communicating artery aneurysm presenting with vision loss. J Neurosci Rural Pract. 2013;4(3):305-7.

2 Lauritzen M. Pathophysiology of the migraine aura. The spreading depression theory Brain. 1994;117:199-210.

3 Shams PN, Plant GT. Migraine-like visual aura due to focal cerebral lesions: case series and review. Sur Ophthalmol. 2011;56(2):135-61.

4 Hoshina Y, Fallah S, Baker V. Optic neuritis mimicking migraine with scintillating scotoma. Am J Med. 2021. https://doi.org/10.1016/j.amjmed. 2021.04.040.

5 Park JH, Park SK, Kim TH, Shin JJ, Shin HS, Hwang YS. Anterior communicating artery aneurysm related to visual symptoms. J Korean Neurosurg Soc. 2009;46(3):232-8.

6 Lee K, Shin SK, Park SH. Acute retrobulbar optic neuropathy as the sole manifestation of subarachnoid haemorrhage from a ruptured anterior communicating artery Aneurysm. Neuroophthalmology. 2013;37(4):172-4.

\section{Submit your manuscript to a SpringerOpen ${ }^{\odot}$ journal and benefit from:}

- Convenient online submission

- Rigorous peer review

- Open access: articles freely available online

- High visibility within the field

- Retaining the copyright to your article

Submit your next manuscript at $\boldsymbol{\nabla}$ springeropen.com 